\section{Granular Packing Under Vibration}

\section{Eric Clément, Laurent Labous and Loic Vanel, Université Pierre et Marie Curie, Paris}

When vibrated, assemblies of grains exhibit a very rich phenomenology. The material - a versatile state of matter itself - can behave in ways reminiscent of solids, of gases or of fluids. There are even signs of unexpected motion

What the lazy physicist has for breakfast is instant coffee, made from grains, and a cereal like cornflakes. He or she buys the coffee as a dry powder, and the cereal as a dry grain. Both are processed in clever ways by their manufacturer, almost certainly involving, at some stage, vibration.

In fact many practical industrial situations involve the vibration of powders and granulates as an important part of a fabrication process. Mechanical agitation is used to transport grains along conveyor belts or to sort granular assemblies via devices using sifting or spontaneous sizesegregation effects. This can be seen as a natural occurrence in mixtures of commercial cocktail nuts shaken during transportation: you always see the larger nuts on top. But this spontaneous size segregation due to shaking, or any other mechanical action like shearing, is a nuisance when the aim is to obtain properly mixed particulate materials. For example, the manufacturing of homogeneous pharmaceutical tablets out of various powdered chemicals is a headache for engineers. Segregation must also be mastered to produce the new highly resistant concrete materials, made of solid particles ranging from 100 Angstroms to a few millimetres in size.

For some applications, vibration is used to obtain a fluid-like matter, but interestingly, vibration is also used to obtain a more solid-like matter such as compacted iron casting that uses this effect. Also, in the making of all ceramics the compaction phase, usually achieved by vibration, is an essential step that ensures the future mechanical strength of the material. This type of technology, developed by engineers through the years, uses the extremely versatile and ubiquitous properties of granular assemblies whose apparent behaviour may depend critically on the nature of the grains, the presence of a surrounding gas, the shape of the container boundaries and the modes of vibration.

Recently, these issues were addressed at a more fundamental level by physicists trying to understand the basic mechanism. How can mass, momentum, and energy transfer from a vibrating boundary, explain the rich variety of behaviour found? Our aim is to give an overview of various phenomena that may take place when an assembly of grains is shaken. We packings. We will describe a process of

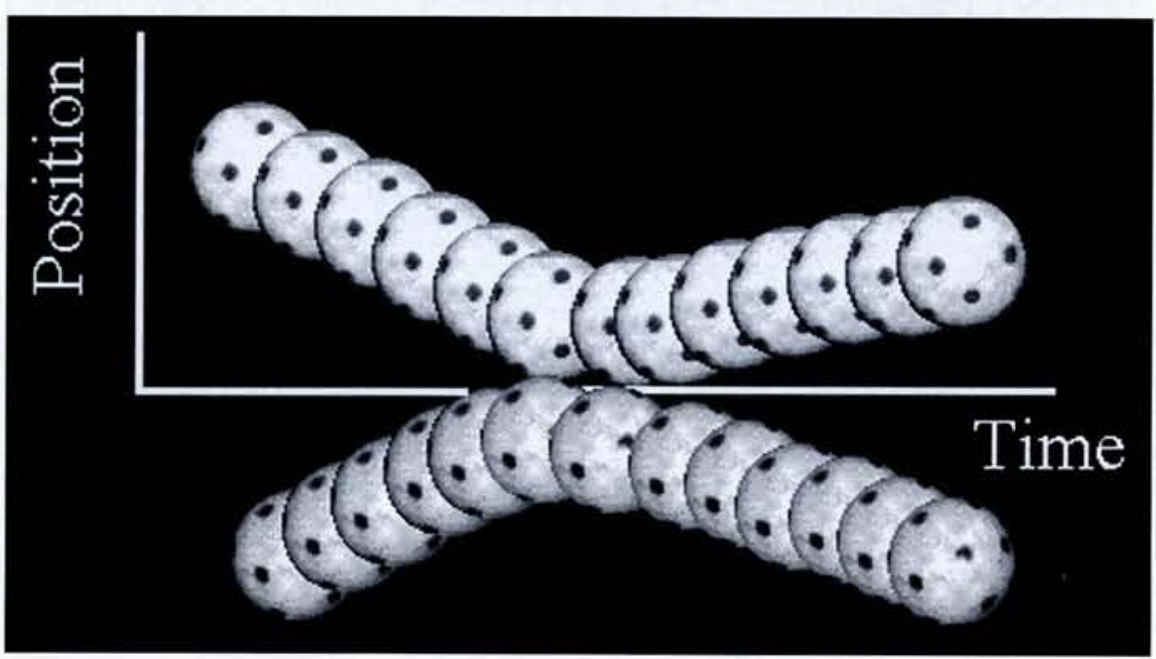

shall show that some minimal concepts and a very crude model of local granular interactions demonstrate many of the key features.

Since what happens is so diverse, we shall only discuss granular material subject to a vertical vibration, with no other interactions, other than solid on solid contact forces. In particular, we ignore the effect of a surrounding gas, even though it can be very important in practice, especially when the size of the grain is small, of the order of 100 micrometers or less. We shall also ignore adhesion forces, whether from the van der Waals forces or from the liquid bridges created by humidity. This means that we focus on situations involving millimetre-sized dry grains, rather than micrometric-sized powders.

First we must discuss the problem of local energy dissipation and its implications for a collection of grains. We can then assess situations where the grains are subject to a vertical vibration. It will emerge that, in different conditions, the grain behave as a solid block, as a gas, or as a condensed fluid. As a fluid there can be behaviours such as convection rolls, spontaneous heaping of the surface, the slow dynamics of glassy materials, and standing wave patterns analogous to the surface Faraday instability of fluids. Our level of description of the interactions is not derived from any firm fundamental basis. Unlike molecular physics the interactions will not come from fundamental laws like quantum theory or Maxwell's equations. In practice, we know only crudely the relevant physical interactions occurring during the contact of two macroscopic bodies. Usually the collisional interaction between spherical grains may be described using two basic quantities, such as a normal and a tangential restitution coefficient for the relative velocities before and after an impact. This simple approach could conceal the fact that these coefficients incorporate a series of very complex mechanical phenomena, involving elasticity, and also irreversible processes such as plastic or visco-elastic deformation. Restitution coefficients are not fundamental material properties. They may vary with strength and angle of impact. There are many different models, but the major feature is the local nature of

Fig 1 Irreversible collision of two dissipative (ie energy losing) particles. Due to the dissipative nature of the collision between real granular particles, the time reversibility of the interaction is broken. This peculiar feature of granular materials is the origin of much complex behaviour 

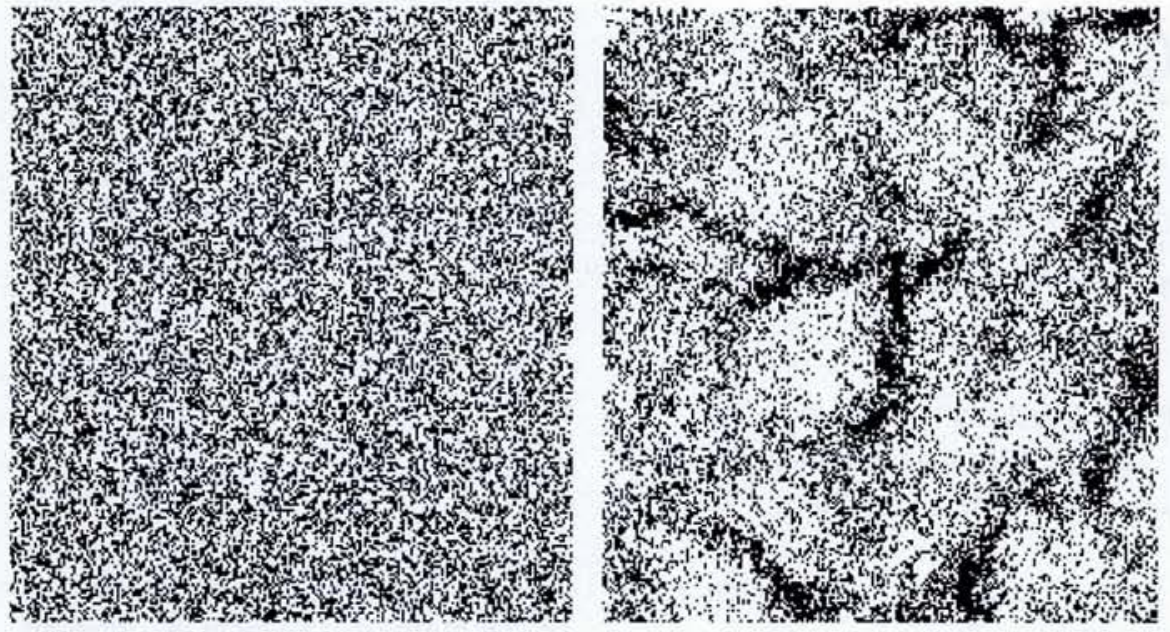

Fig 2 Cooling of a 'granular gas'. Numerical simulation of a hard sphere dissipative gas. Fig 2 a left An initial homogeneous configuration with a random velocity distribution. Fig $\mathbf{2 b}$ bight After about 20 collisions per particle dense clusters form due to local energy dissipation. These clusters grow because they are low pressure regions

dissipation. Already one has a situation radically different from the standard molecular dynamic vision of kinetic theory. In the classical kinetic approach, time reversibility and energy conservation of the local interactions are fundamental ingredients from which one can derive nice properties such as the $\mathrm{H}$-theorem, and establish a rigorous route to macroscopic thermodynamic laws. These features disappear with local dissipative interactions.

When two macroscopic bodies collide

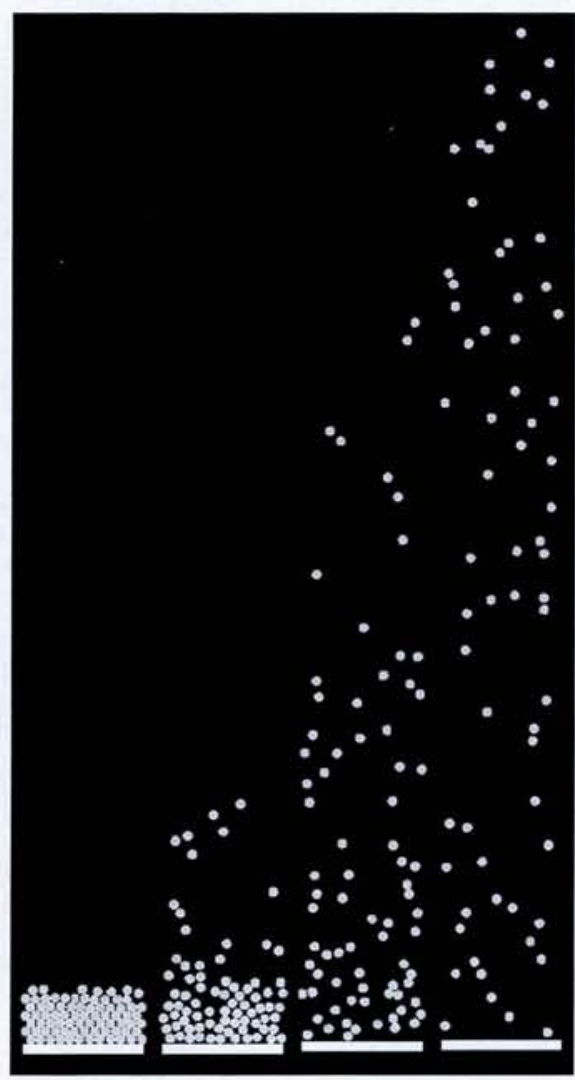

(figure 1) it is always possible to decide which is the past and which is the future of the collision, from knowledge of the trajectories. Roughly, one may say that local dissipation tends to guide the trajectories of two colliding bodies to approach each other after each impact. This implies a new situation (figure 2 ), which shows the results of a numerical simulation using a simplified dissipation model. The normal restitution coefficient is $e=0.6$ ( $e$ is the normal relative velocity after the collision, divided by the normal relative velocity before the collision). The simulation starts with the initial 'big-bang' situation shown in figure $\mathbf{2 a}$ - particles distributed on a surface with random positions and velocities. After several collisions per particle an instability is observed which is directly linked to the local dissipation mechanism. Figure $2 \mathrm{~b}$ shows that the 'cooling' of the granular assembly is not homogeneous. Regions of higher densities are regions of lower pressure and lower 'temperature'; they have the tendency to attract more particles. In this situation the only available energy sources are the spontaneous shearing fluctuations generally present in any fluid-like system. The soft-clustering mode shown is the result of a subtle competition between cooling and heating. It was noticed first by a team at the University of Tel Aviv (I. Goldhirsh and G. Zanetti Phys. Rev. Lett. 70 (1993) 1619).

Another important characteristic of assemblies of grains is the existence of a finite dissipation length. When a moving boundary gives energy to the grain assembly, the collision wave caused is damped exponentially with a length proportional to the size of the grains. The numerical pre-factor which sets this length scale, increases when the dissipation decreases. For granular assemblies which are bigger than this dissipation length, what happens bears some analogy with a phase transition. Starting from a packing where the grains are separated, and then after the passage of the collision wave, there are dense zones characterized by binary colli-

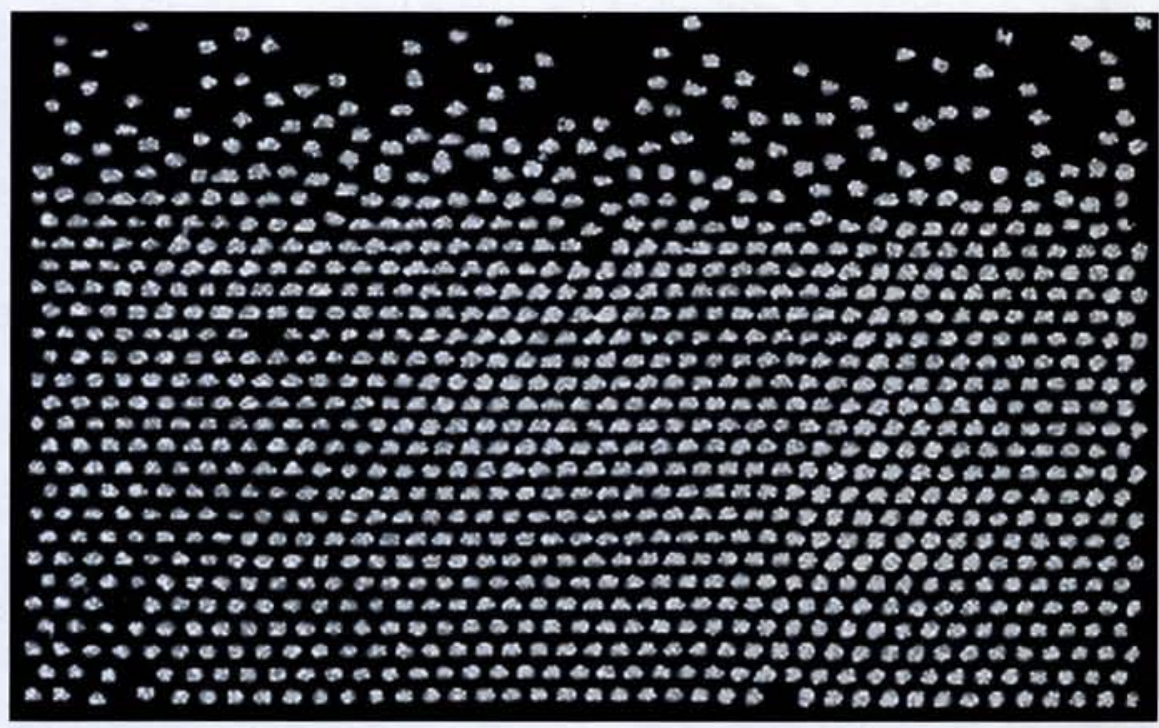

Fig 3 Vibrofluidization of a granular packing

Fig 3a left Submitted to a vertical vibration a thin layer of weakly dissipative grains will expand when the input energy is large. From left to right, the pictures are the result of simulations for increasing vibration amplitude. In the last picture the granular column reaches a steady state resembling a kinetic gas; this is the so-called 'fluidized state' Fig $3 \mathbf{b}$ above Thicker layers of grains dissipate so much energy that they cannot be entirely fluidized. Taken from an experiment in a bidimensional cell this snapshot shows that only a few grains on the top surface are fluidized while the remaining grains move as one block 
sions whose number increases tremendously. In numerical simulation this number of collisions would diverge in a finite time. In practice this means that the system will reach a state where the particles undergo long-lasting and multiple contacts: they cannot be described by independent binary collisions any more. The granular assembly behaves more or less like a compact solid or a disordered glassy material.

When rate of energy input is large enough to prevent excessive 'cooling' and when the height of the layer is small with respect to the dissipation length, a steady state is reached. The assembly looks like a gaseous column, the 'fluidized state', which has been studied in parallel by many teams in Paris, in Germany and in Cambridge. Figure $3 \mathrm{a}$, shows the results of a numerical simulation of a weakly dissipative and shallow granular assembly, excited by a sinusoidally driven vibrating plate. As the acceleration is increased, a fluidized state develops, and the assembly. resembles a gas column. If the number of bead layers is increased, a novel state is reached. The fluidization wave arising from collisions with the bottom plate is almost damped on a time scale comparable to the period of vibration. The system separates into two phases: at the top is a dense fluid (figure 3b); at the bottom, a solid-like structure appears. The regular triangular packing of the solid structure of figure $3 \mathrm{~b}$ is due to two-dimensional confinement of the metal beads used in the experiment. In a three-dimensional situation, the appearance would be more like amorphous packing.

Finally, when the height of the layer is much larger than the dissipation length, all the input energy is dissipated in the bulk, and the system behaves like a solid inelastic block on a vibrating plate. To a very good approximation all the energy received from the impact is immediately dissipated in the bulk and the granular assembly velocity adjusts to the plate velocity. In this case, the granular layer may move more or less like a distorting snake. This is the so-called sub-harmonic instability found by a team at the Ecole Normale Supérieure in Lyon, France. If the driving acceleration is very large, the solid block is launched with such a high velocity after each collision that it undergoes a free flight lasting at least one full period of vibration. Due to the initial disorder of the granular assembly and friction with the wall, different parts of the system may respond with different phases. When one
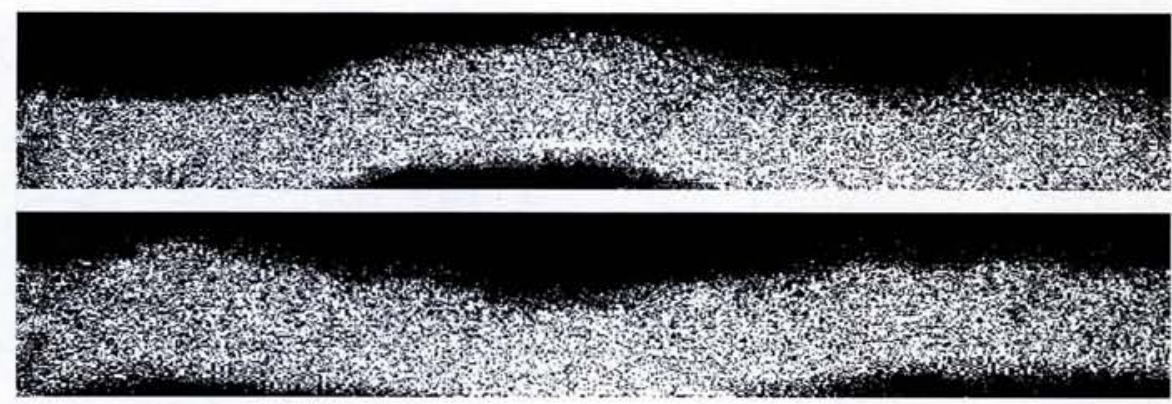

Fig 4 During a vibration cycle, a granular layer may detach from the floor and perform a free flight. When the time of free flight becomes larger than the vibration period, an horizontal thick layer may become unstable. This is the socalled 'sub-harmonic instability'. The top picture displays an experimental snapshot of the instability. The bottom picture is a snapshot one period of vibration later

part touches the plate another part can still be in the middle of a free flight. This is the case for the top part of the snapshot displayed in figure 4 where the left and right parts of the layer touch the bottom plate and where the middle part is still in the air. One period later the opposite situation is observed (see bottom part of figure 4 ). The packing responds like an inelastic block with the different parts separated by a phase defect which decreases with time. Ultimately the system will respond as a single inelastic solid block.

The dense state is the most complex and versatile situation. We have mentioned already the sub-harmonic instability which happens for rather large horizontal layers. When the aspect ratio is closer to one (more like a square), if the friction between the grains and the lateral walls is important, another phenomenon predominates. For sinusoidal vibrations with an acceleration larger than gravity the motion of a solid inelastic block on a plate separates into two distinct phases. One is a free flight phase where the solid block leaves the plate and performs a free flight. The other is a contact phase, initiated by a collision between the packing and the plate. At the beginning of the free flight there is a moment when the velocity of the granular layer is directed upwards but the lateral boundary goes downwards, and the ballistic motion of the layer is damped due to friction. Actually, this contrary motion is the origin of a singular boundary layer effect which results in two symmetrical convection rolls. This effect was found and studied by a group at the University of Paris, and also later at the University of Chicago, US. For example, when an horizontal layer of sand is vibrated in a container with vertical boundaries, a surface instability is observed. The instability is directly caused by two convection rolls on each side, which bring grains downwards along the walls and upwards in the centre of the bulk (figure 5). This difference of fluxes creates heaps on the free surface,

Fig 5 Boundaries such as the lateral walls are responsible for the existence of convection rolls which bring materials upwards at the centre of the layer and downwards at the side. As shown by this experimental picture, the surface may become unstable and form a heap where the mass transfer mechanism is a continuous avalanche; this contributes to the flow

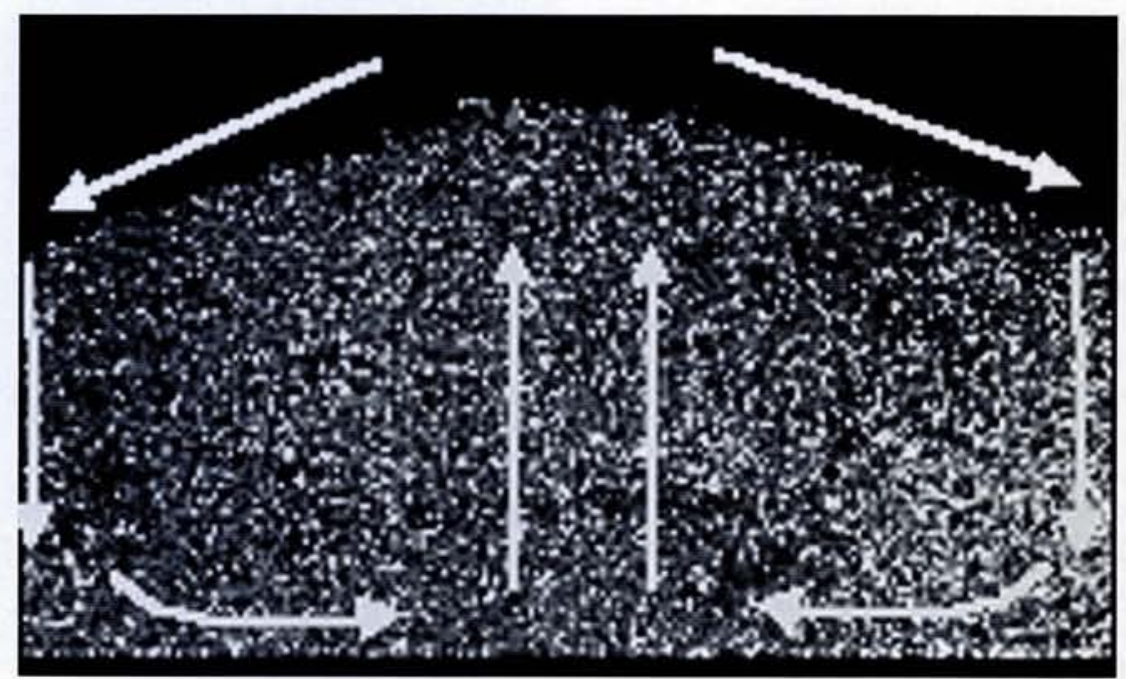


which show that the state is not really fluid (a heap could not exist) nor really solid (convection rolls would be absent).

At higher vibrational energy, the local agitation of the grains increases. The heap structure is mechanically unstable and, eventually, an horizontal free surface is restored. In this case, the flow of the grains really is akin to a fluid. This property is used in the industrial process of iron casting. When a fluid-like state has been achieved by mechanical agitation of a sand container, a polystyrene replica of the piece to be moulded is plunged in the sand bed in such a way that when the vibration stops the piece is surrounded homogeneously by the sand. The aim of the next stage is to cast a hot liquid metal, which burns out and replaces the polystyrene piece. Any vapour escapes easily through the porous sand. A successful procedure needs a sand matrix of high mechanical strength, able to resist large stress gradients during the cooling phase of the metal. The fluidized bed, which settles in its rather loose packing once vibration has stopped, does not have this property. So, before casting the liquid metal the granular assembly is again vibrated, but in a different parameter range. The system compacts itself forming a dense and solid structure, very resistant to compression, but still very easy to remove by toppling the container, after the metal piece has cooled.

The compaction process has recently shown links to more fundamental behaviour. Physicists at the University of Chicago, US, studied the density variation in a long vertical column of grains submitted to a series of vertical taps. There was an initial fast compaction stage. After a short time slow dynamical behaviour emerged, characterized by a logarithmic increase of the density (figure 6). This logarithmic variation could last several days before reaching a steady state. The underlying idea is: to get an additional increase in density when the system is already very packed it is necessary to have a collective reorganization of large parts of the packing. This collective phenomenon is exponentially costly in time for particles with independent and random motions. Such behaviour is reminiscent of many processes of ageing in other physical contexts, such as the glass transition. Several models have been proposed which follow analogies with the statistical physics of spin glasses.

Another surprising feature of verticallyvibrated layers of grains is the existence of

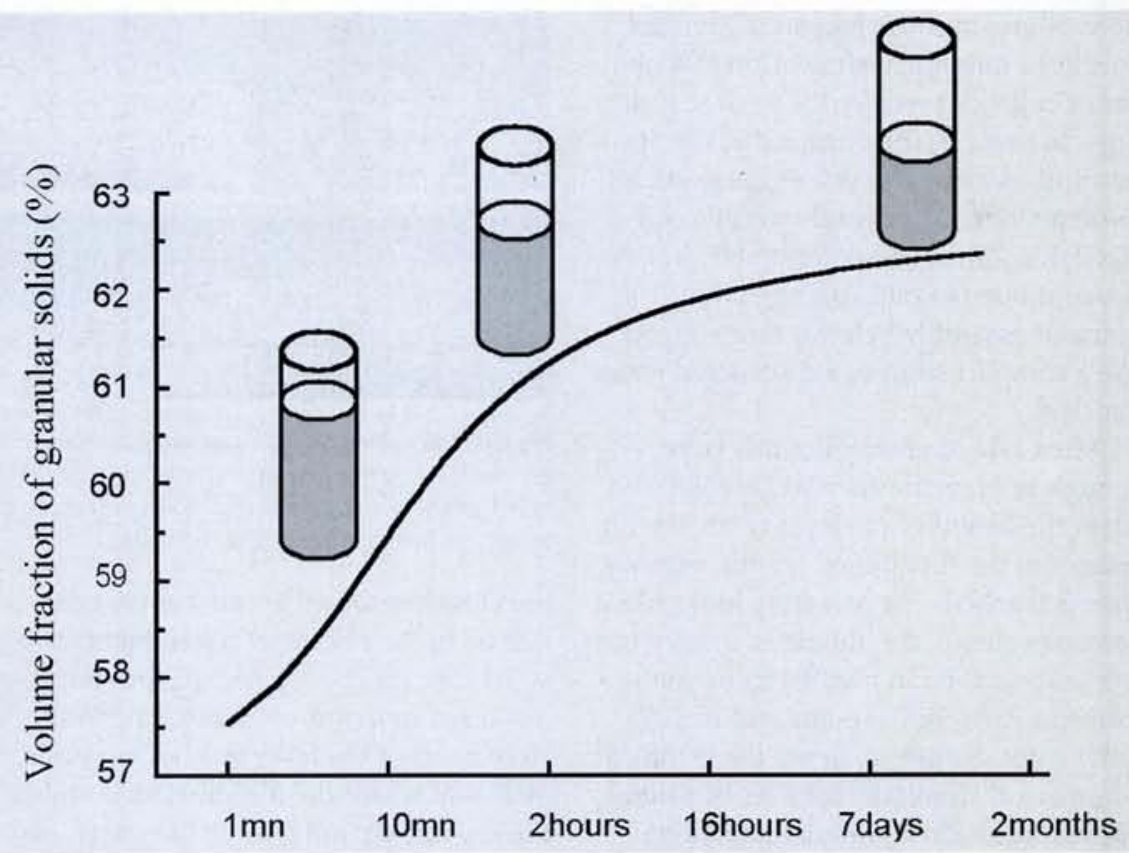

Fig 6 above By choosing suitable vibration parameters, it is possible to make a granular column more and more compact. In a given volume, the percentage of granular solids increases with time, at first rapidly, but thereafter it takes a very long time to reach a final density. Mainly, the compaction process is logarithmic in time

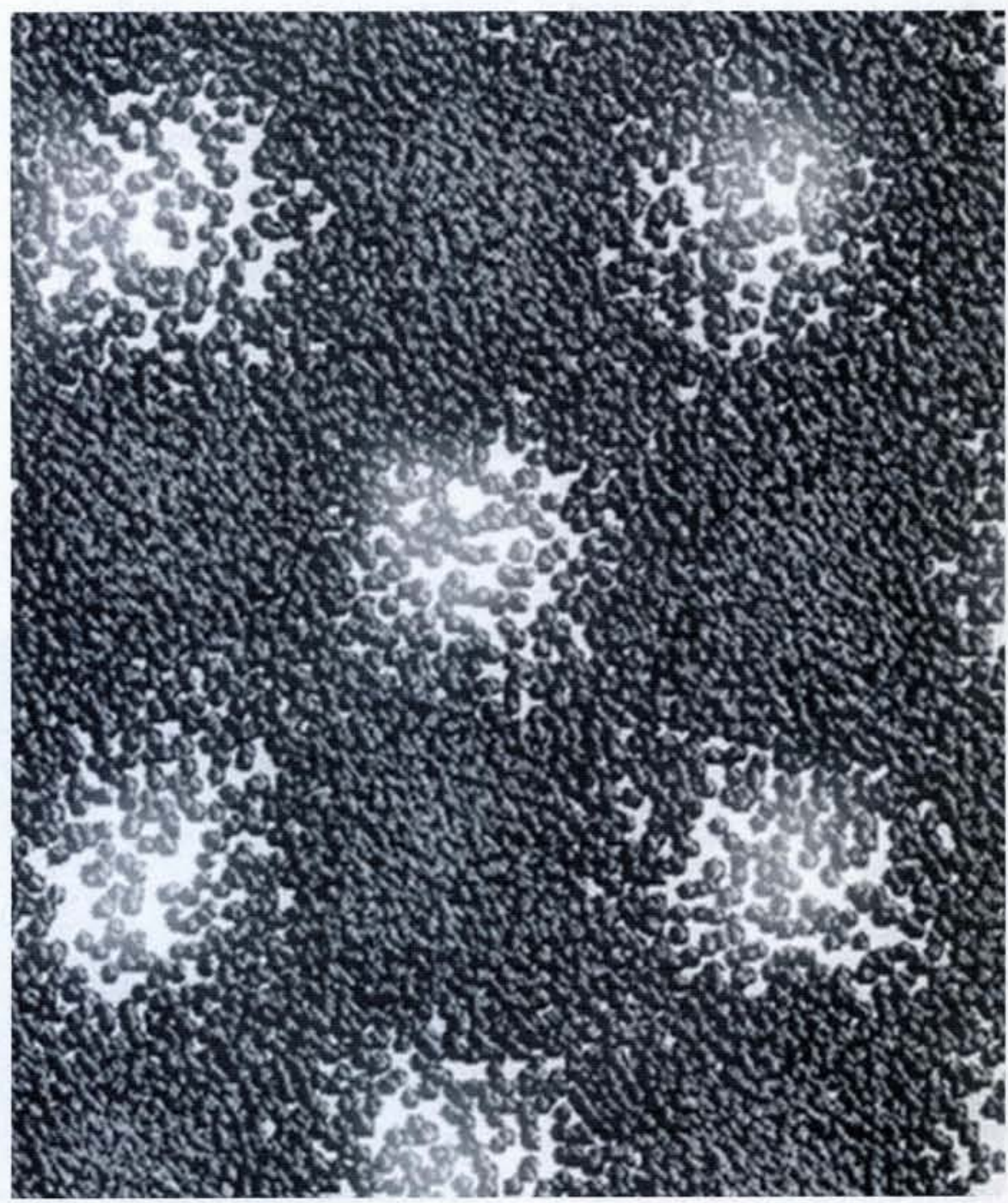




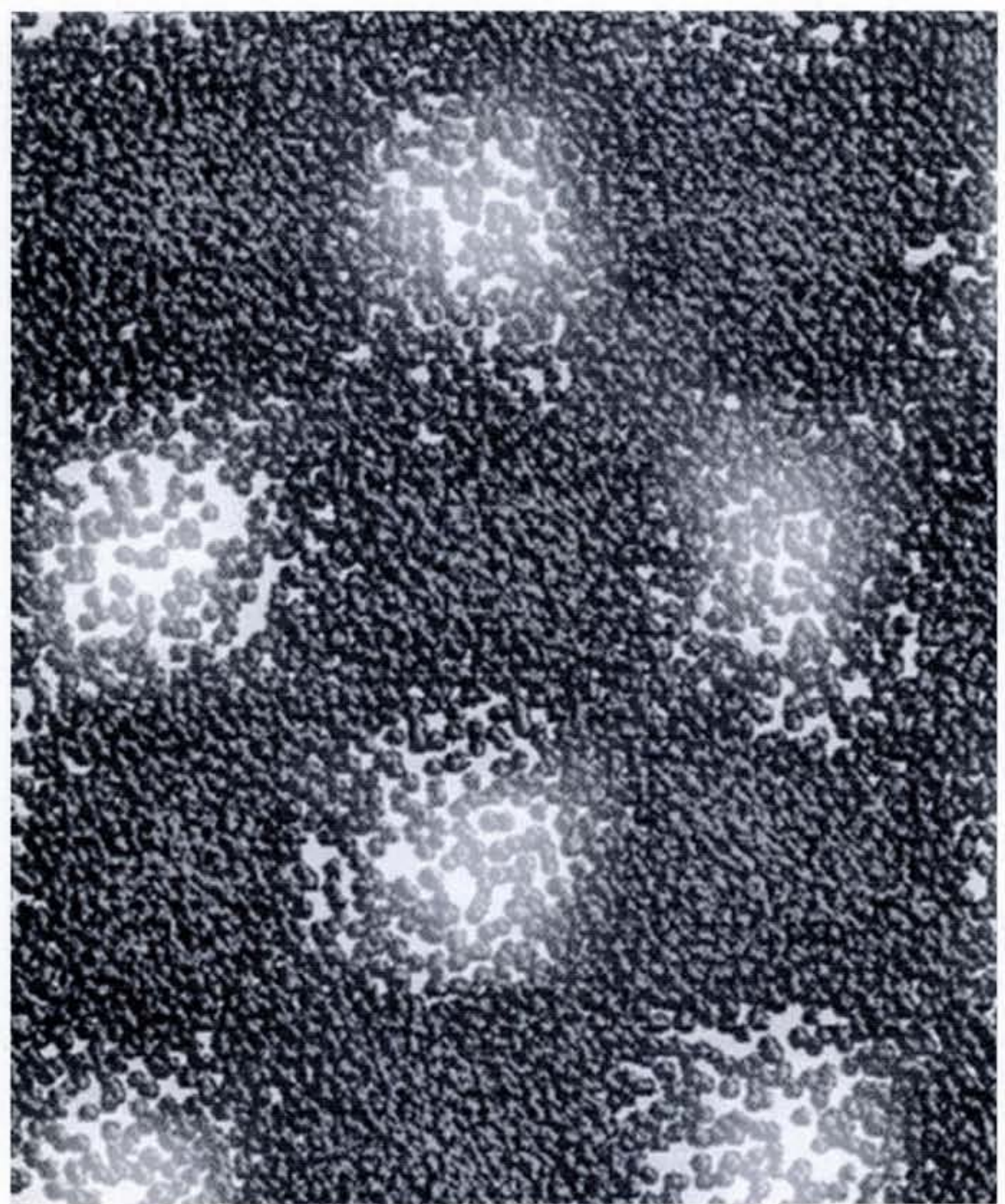

Fig 7 left and above The Faraday instability in granular assemblies. A numerical simulation of square patterns as observed from the top of a vertically vibrated container. Snapshots left and above are separated in time by one period of the excitation showing the exchange of peaks and cavities from one period to the next. This phenomenon is well know in fluids and was discovered by Michael Faraday in 1831

Fig 8 below The Faraday standing wave pattern in a bidimensional cell.

Fig $8 \mathbf{a}$ is an experimental snapshot of a standing wave for a layer of aluminium beads confined in a bidimensional cell. A vertical cut of a pattern (as in fig 7) would display such a structure. Fig $8 \mathbf{b}$ is the snapshot of an 'oscillon', which is a solitary peak pattern occurring at the edge of the Faraday instability
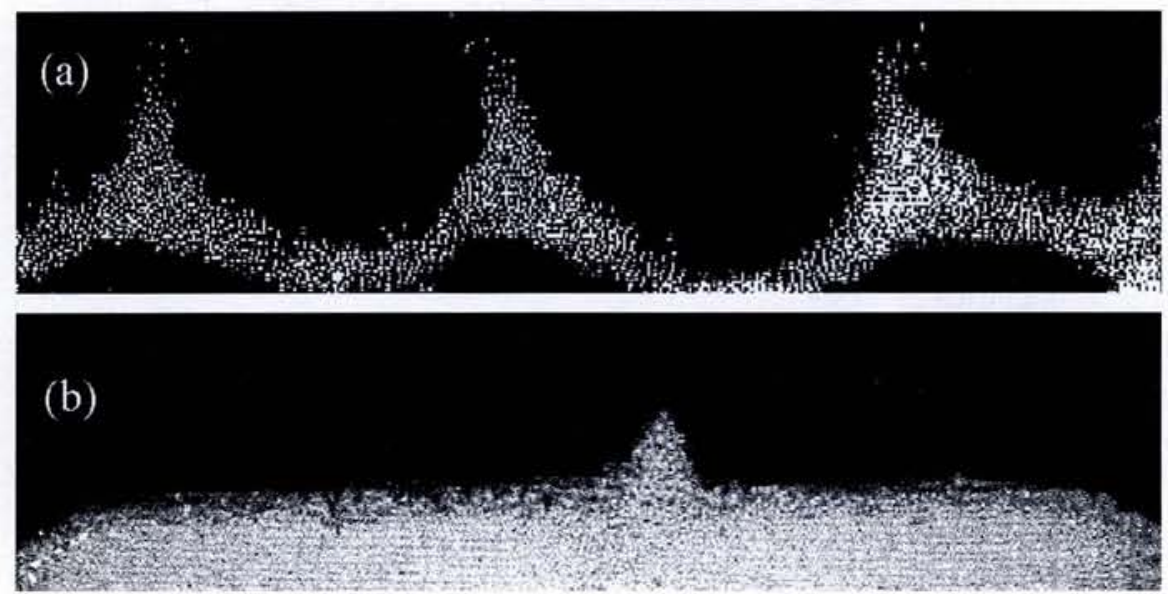

resonant standing waves. These were first discovered by physicists in Lyon, France, and observed more recently in a different geometry at the University of Texas, US. The Texas experiment shows that, when subject to vertical vibration, a thin layer of grains on an horizontal plate may produce various resonant patterns. These include squares, stripes and even hexagons. We reproduce (figure 7 ) the results of a computer simulation of this behaviour, for a layer of 6 grains vibrated sinusoidally at an acceleration amplitude of 3.2 times the acceleration of gravity. These views, from above, show two successive phases separated by a period equal to the period of excitation. The system displays a square structure. It is strikingly reminiscent of the surface instabilities observed last century by Michael Faraday for vibrated layers of fluids. The phenomenon results from parametric excitation of gravity waves or capillary surface waves, and the patterns seen result from non-linear couplings between waves travelling in different directions. The Texas group has recently reported the formation of an original localized structure which they have baptized the 'oscillon'. It occurs just above the threshold of instability. This singular oscillating structure is formed alternately from a peak and a hole.

Surprisingly, it is possible to create situations where several of these 'oscillon' structures couple to form dimers, trimers or even higher order 'molecules'. In figure $8 \mathrm{a}$ we show a snapshot of an experiment on a confined bidimensional layer of aluminum beads. The top picture shows a snapshot of a resonant wave; this is a lowdimensional analog of the Faraday instability, and corresponds to a vertical cut of a picture like figure 7 . The lower picture, figure $8 \mathrm{~b}$, shows an oscillon localized at the centre of the cell. A full understanding of these waves is still lacking. There are open problems and challenging questions on the many fundamental and unveiled aspects of energy and momentum transport in these complex states of matter which can be formed with granular materials.

\section{Further reading}

H.M. Jaeger, S.R. Nagel and R.P.

Behringer Rev. Mod. Phys. 68 (1996) 1259 a review of recent developments in the physics of granular matter

I. Gutman Industrial Use of Mechanical Vibrations (Business Books, London, 1968) applications 